\title{
Transformada de Hilbert-Huang Modificada com Redução do Ruído para Sinais de Sonar Passivo
}

\author{
Elio P. Sarno Filho, Anderson D. Santos, Eduardo F. Simas Filho, \\ Antônio C. L. Fernandes Jr. e José M. de Seixas
}

\begin{abstract}
Resumo-A transformada de Hilbert-Huang (Hilbert-Huang transform - HHT) é uma técnica de análise tempo-frequência (time-frequency - TF) baseada na decomposição empírica em modos (empirical mode decomposition - EMD), que vem sendo aplicada com sucesso a sinais não-estacionários e sistemas nãolineares, entre os quais, os sinais de SONAR passivo. No entanto, o desempenho da HHT/EMD pode ser bastante degradado em condições de baixa relação sinal-ruído (signal-to-noise ratio SNR). O presente trabalho propõe um método de análise TF para sinais de SONAR passivo baseado na HHT/EMD, no qual a redução de ruído é realizada a partir da análise da função de autocorrelação (ACF) e da filtragem wavelet com limiar (WTD), executados após a EMD. Os resultados obtidos com sinais experimentais de SONAR passivo são utilizados para validar o método proposto.
\end{abstract}

Palavras-Chave-HHT, EMD, SONAR passivo, ACF.

Abstract-The Hilbert-Huang transform (HHT) is a timefrequency (TF) analysis technique based on empirical mode decomposition (EMD), which has been successfully applied to non-stationary signals and non-linear systems, including passive SONAR signals. However, the performance of HHT/EMD can be greatly degraded under conditions of low signal-to-noise ratio (SNR). The present work proposes a TF analysis method for passive SONAR signals based on HHT/EMD, in which noise reduction is performed by analyzing the autocorrelation function (ACF) and the wavelet threshold denoising (WTD), performed after EMD. The results obtained with experimental passive SONAR signals are used to validate the proposed method.

Keywords-HHT, EMD, Passive SONAR, ACF.

\section{INTRODUÇÃO}

Nas últimas décadas, tem-se observado um grande avanço na área de processamento de sinais acústicos submarinos (Underwater Acoustic Signal - UAS), notadamente na utilização de sistemas de navegação e determinação da distância pelo som (SOund NAvigation and Ranging - SONAR) com finalidades civis, a exemplo do monitoramento do ambiente e da fauna submarinos, e com propósitos militares. Neste último caso, destaca-se a utilização do SONAR passivo em operações submarinas, cujos principais objetivos são detectar, classificar e localizar embarcações, evitando sua própria detecção [1].

Visando otimizar o desempenho do SONAR passivo, vários algoritmos de análise de UAS tem sido propostos, a maioria deles baseados na transformada de Fourier de tempo curto (short-time Fourier transform - STFT) [2], dada a aplicabilidade desse método de análise TF a sistemas não-lineares e sinais não-estacionários, tais como os de SONAR passivo [3].

Elio P. Sarno Filho, Anderson Damacena Santos, Eduardo F. Simas Filho e Antonio C. L. Fernandes Jr., PPGE/UFBA, Salvador-BA, Brazil. E-mails: \{elio.pithon, anderson.santos, eduardo.simas, antonio.fernandes\} @ufba.br,

José M. de Seixas, LPS/PEE/COPPE/POLI/UFRJ, Rio de Janeiro-RJ, Brasil. E-mail: seixas@lps.ufrj.br.
Uma das técnicas tradicionalmente utilizadas no processamento de sinais de SONAR passivo, que se baseia na STFT, é a análise demodulação da envoltória de modulação no ruído (Demodulation of Envelope MOdulation on Noise DEMON) [4], apresentada na Fig.1. Após seleção, o sinal $s(t)$ é filtrado para limitação de banda e demodulado pela detecção da envoltória do ruído de cavitação [3] gerado pelo hélice de embarcações, cujo conteúdo espectral específico fornece informações da velocidade de rotação do eixo e número de pás desse hélice. Essas informações permitem identificar e classificar o tipo de propulsão da embarcação [3] [4].

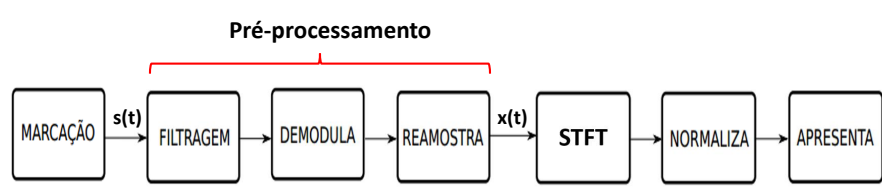

Fig. 1: Diagrama de blocos da análise DEMON padrão.

Apesar do bom desempenho da STFT na análise espectral de UAS, essa técnica possui limitações, sendo a principal delas, a necessidade do tamanho da janela temporal ser compatível com o conteúdo espectral do sinal analisado, a priori desconhecido. Uma janela muito estreita provoca espalhamento espectral e consequente diminuição da resolução frequencial [5]. Visando superar essa limitação, a HHT/EMD [6] vem sendo cada vez mais utilizada na análise de UAS, por permitir a representação de sinais não-estacionários e sistemas nãolineares sem necessidade de janelamento [6].

A literatura técnica vem reportando o uso da HHT/EMD em diversas aplicações. $\mathrm{Na}$ área médica, por exemplo, quando combinada com uma rede neural convolucional profunda $(\mathrm{CNN})$, tem sido usada em sistemas de imagens médicas rápidos e eficientes [7]. Um preditor de radiação solar diária, que integra a EMD a uma rede neural de retro-propagação (BPNN), obteve uma maior eficiência em sistemas fotovoltaicos inteligentes [8]. Também vem sendo utilizada no diagnóstico de falhas potenciais em máquinas industriais [9] e no processamento de sinais acústicos [10].

Embora a HHT/EMD possa ser aplicada de forma eficiente em UAS, o seu desempenho geralmente é prejudicado pela contaminação do sinal de interesse por fontes de ruído ambiental [11]. Assim sendo, um método de análise TF de sinais de SONAR passivo com redução de ruído (HHT-modificada) é proposto no presente trabalho. Nessa nova estratégia, as funções de modo intrínseco (intrinsic mode function - IMF), resultantes da decomposição UPEMD (variante da EMD), são classificadas como IMF-R (contendo apenas ruído) ou 
IMF-T (IMF de transição, composta de sinal de interesse e ruído), com base no valor da função de autocorrelação (autocorrelation function - ACF) [12]. Após a separação, as IMF-R são descartadas e as IMF-T são tratadas pela filtragem wavelet com limiar (wavelet threshold denoising - WTD) para redução de ruído [13]. Os resultados obtidos na aplicação do novo método a sinais experimentais de SONAR passivo indicam um melhor desempenho em relação à análise DEMON padrão, com respeito a uma maior resolução espectral.

\section{DECOMPOSIÇÃO EMPÍRICA EM MODOS E TRANSFORMADA HILBERT-HUANG}

A HHT é um método empírico de análise de sinais cuja base de expansão é adaptativa, de forma a produzir uma representação fisicamente significativa de sistemas não-lineares e sinais não-estacionários [6]. Como mostra a Fig.2, a HHT é composta das seguintes etapas:

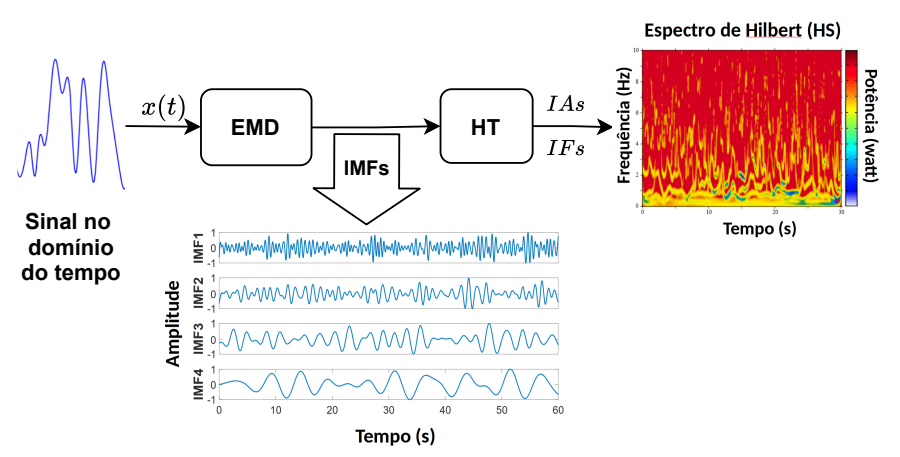

Fig. 2: Diagrama de blocos da transformada Hilbert-Huang (HHT).

(i) Decomposição pela EMD do sinal-alvo em um conjunto de componentes ortogonais de média nula, as IMFs [6];

(ii) Aplicação da transformada de Hilbert (Hilbert transform - HT) [2] a cada IMF;

(iii) Estimação do espectro de Hilbert (Hilbert spectrum HS) [6] a partir da informação tempo-frequência-energia extraída das IMFs.

A EMD é um método adaptativo de decomposição de sinais, cuja essência é identificar e extrair os modos intrínsecos de oscilação contidos em um sinal e, a partir deles, decompor esse sinal em um conjunto de componentes com amplitude e frequência variáveis (IMFs) [6]. As IMFs são obtidas por um processo iterativo conhecido como "peneiração" (sifting), em que as assimetrias entre as envoltórias superior e inferior do sinal original $x(t)$, são progressivamente removidas [6].

Uma vez obtido o conjunto de IMFs, as amplitudes instantâneas (instantaneous amplitude - IA) e frequências instantâneas (instantaneous frequency - IF) [6] são estimadas pela aplicação da HT a esse conjunto. Por fim, determina-se o HS associado a $x(t)$, pela superposição das contribuições individuais das IAs e IFs referentes às IMFs [5]. A partir do HS, é possível se obter o espectro marginal de Hilbert (marginal Hilbert spectrum MHS), que representa a amplitude total de cada componente IF, acumulada no intervalo de duração do sinal $x(t)$ [6].

A EMD pode apresentar limitações em aplicações práticas, sendo a mixagem de modos (mode mixing - MM) [14] a maior delas. A MM decorre da forma como as IMFs são geradas no processo de "peneiração", que pode falhar no caso de sinais intermitentes, sinais com componentes de frequência muito próximos (com um fator multiplicativo menor ou igual a 2 entre eles) ou sinais muito ruidosos [14].

Vários algoritmos baseados na EMD tem sido propostos para reduzir o MM e a sensibilidade ao ruído [15], entre os quais pode-se destacar a decomposição empírica do conjunto em modos (ensemble empirical mode decomposition - EEMD) e a decomposição empírica em modos com fase uniforme (uniform phase empirical mode decomposition - UPEMD). O primeiro é uma modificação da EMD, que consiste em adicionar ruído branco Gaussiano (white Gaussian noise - WGN) ao sinal de interesse [14]. A UPEMD é uma variação da EEMD em que o ruído WGN é substituído por um sinal senoidal. Enquanto a EEMD soluciona o MM apenas nos casos de sinais intermitentes, a UPEMD também apresenta bom desempenho em se tratando de sinais com componentes de frequência muito próximos [15], tais como os sinais de SONAR passivo. Portanto, teoricamente, a UPEMD é o método mais adequado para tratar esse tipo de sinal.

\section{TRANSFORMADA DE HILBERT-HUANG MODIFICADA}

O desempenho da EMD pode se degradar nos casos de baixa SNR, em que o sinal-alvo e o ruído ocupam a mesma banda de frequência, tais como os sinais de SONAR passivo. Nesses casos, a redução de ruído pode se tornar impossível pelos métodos de filtragem convencionais [11]. A Fig.3 apresenta as etapas da HHT-modificada, proposta neste trabalho, para redução de ruído em SONAR passivo.

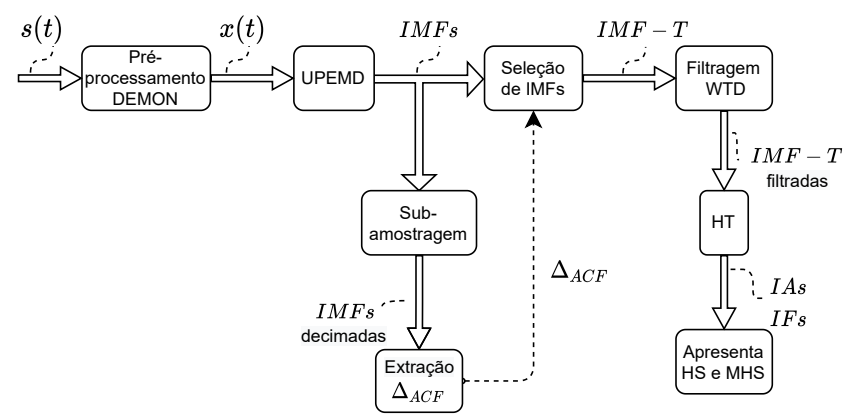

Fig. 3: Diagrama de blocos da HHT-modificada.

Inicialmente o sinal SONAR $s(t)$ é pré-processado pela análise DEMON (Fig.1), obtendo-se $x(t)$. Em seguida, é aplicada a decomposição UPEMD e são obtidas as cinco primeiras IMFs, nas quais estão localizados os componentes harmônicos significativos dos sinais de SONAR passivo.

Um processo de subamostragem das IMFs, para que a banda de frequência (originalmente estreita) de cada uma delas passe a ocupar toda faixa útil $[0-\pi]$ [2], é realizado apenas para o cálculo dos coeficientes ACF, sem interferir no processamento propriamente dito dessas IMFs. A ACF mede a similaridade entre as amostras de uma série temporal, em 
função do intervalo $\eta$ (lag) entre elas, sendo definida por [12]:

$$
r_{x x}(\eta)=\sum_{i=-\infty}^{\infty} x(i) x(i+\eta), \quad \eta=1,2, \ldots
$$

Quando é multiplicada por um fator de normalização $\lambda=1 / r_{x x}(0)$, de forma a assumir o valor 1 para $\eta=0$, passa a ser referida como coeficiente ACF [12]:

$$
\rho_{x x}(\eta)=\frac{r_{x x}(\eta)}{r_{x x}(0)}, \quad-1 \leq \rho_{x x}(\eta) \leq 1
$$

Utilizando uma estrutura de quadros em cada IMF, calculase $\rho_{x x}(\eta)$ de cada quadro, em uma faixa $\left[\eta_{\min }: \eta_{\max }\right]$ empiricamente determinada. Os sinais que tem certa regularidade apresentam $\left|\rho_{x x}(\eta)\right|>0$, enquanto sinais com baixa SNR ou em ruído puro $\left|\rho_{x x}(\eta)\right| \simeq 0$. Em seguida, para identificar as IMF-R, determina-se a mediana cada $\eta$ e o intervalo interquartil $\Delta_{A C F}$ (diferença entre o $1^{\circ}$ e o $3^{\circ}$ quartis) da distribuição das medianas.

O parâmetro $\Delta_{A C F}$ mostrou-se efetivo para a discriminação entre IMF-T e IMF-R, então um patamar de classificação $\Delta_{L}$ foi definido de modo que, quando $\Delta_{A C F}>\Delta_{L}$ a IMF é classificada como IMF-T e, caso contrário, como IMF-R:

$$
\Delta_{A C F} \lessgtr_{I M F-T}^{I M F-R} \Delta_{L}
$$

As IMF-R são eliminadas e é realizada uma filtragem WTD das IMF-T, seguida pela aplicação da HT.

O limiar $\Delta_{L}$ usado neste trabalho foi definido pela aplicação do método proposto a um conjunto de 40 sinais de SONAR passivo. A sua escolha foi feita a partir da análise da curva ROC (Receiver Operating Characteristic - ROC), em que usou-se como critério de eficiência a obtenção de uma taxa mínima de acerto (True Positive Rate - TPR) de 90\% para a classe das IMF-T (classe positiva), valor este considerado suficiente para a preservação das IMFs potencialmente importantes na análise da assinatura espectral das embarcações.

Neste trabalho, para desenvolvimento e validação do método proposto, as IMFs obtidas na decomposição UPEMD foram previamente separadas em duas classes (IMF-T e IMF-R) após análise de um especialista, na qual considerou-se IMF-T aquelas que apresentam pelo menos um componente harmônico com valor de pico $10 \%$ acima do nível médio de ruído de fundo (average noise level - ANL). Deste modo, obtêm-se as classes-alvo para cada IMF.

O processo de escolha de $\Delta_{L}$ se inicia com a aplicação do algoritmo HHT-modificada ao conjunto de 40 sinais de forma sistemática, usando várias configurações de valores dos parâmetros operacionais, quais sejam, o tamanho da janela de análise (window size - WS), o fator de sobreposição entre janelas adjacentes da estrutura de quadros (overlap - OL), o fator de subamostragem das IMFs (decimation factor - DF) e a faixa de LAGs usada no cálculo do coeficiente ACF ([ $\left.\left.\eta_{\min }: \eta_{\max }\right]\right)$. As distribuições dos $\Delta_{A C F}$ relativos às duas classes de IMFs são, então, analisadas por meio da curva ROC, buscando-se localizar o ponto de operação com valores de TPR e da taxa de falsos positivos (False Positive Rate - FPR) que atendam ao critério de eficiência fixado. A partir deste ponto de operação, obtém-se o limiar de separação $\Delta_{L}$ relativo àquela configuração específica de parâmetros operacionais.

\section{RESULTADOS}

Nesta seção, são apresentados os resultados da aplicação da HHT-modificada a sinais experimentais de SONAR passivo. O desempenho do novo método é comparado ao da variante HHT-UPEMD, em que se baseia, como também ao da DEMON padrão. Os sinais-teste e a metodologia utilizados nessa avaliação, são apresentados a seguir.

Um dos sinais utilizados, integra o conjunto de sinais experimentais que foi disponibilizado pelo Instituto Brasileiro de Pesquisa da Marinha (IPqM) [16], tendo sido adquirido na Baía de Guanabara, Rio de Janeiro, por meio de um arranjo de hidrofones que segue uma forma de arco elíptico (Fig.4b e Fig.4c), com frequência de amostragem $F_{a}=31,250 \mathrm{~Hz}$. O cenário de aquisição desses sinais consiste em um navio offshore (usado na operação de plataformas de petróleo), enquanto ele passa ao lado de outro navio menor (Fig.4a).

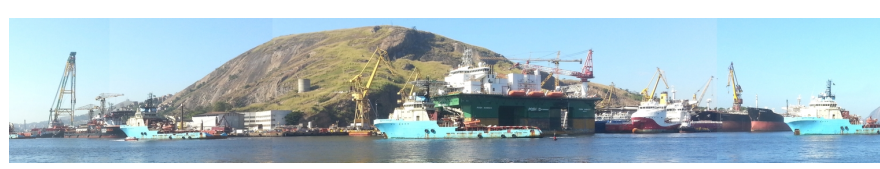

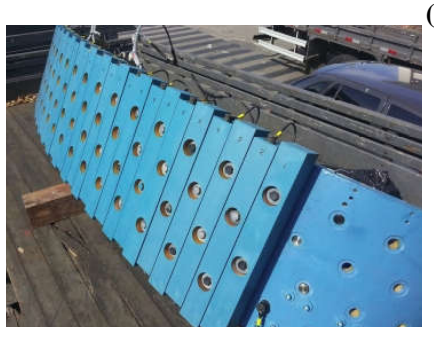

(b)

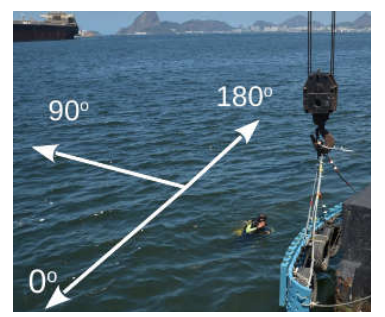

(c)
Fig. 4: Cenário da aquisição de dados usado pela Marinha do Brasil [16].

O segundo conjunto de dados experimentais é composto de 50 sinais do banco de dados acústicos submarinos, adquiridos no porto de Vigo, Espanha [17], com $F_{a}=52,734 \mathrm{~Hz}$. Uma parte desse conjunto, formada por 40 sinais (sub-conjunto de desenvolvimento), foi utilizada na definição do valor de $\Delta_{L}$, enquanto os 10 sinais restantes (sub-conjunto de validação) foram usados na validação desse processo de definição.

Conforme ilustrado na Fig.5, as seguintes medidas quantitativas de desempenho foram utilizadas na avaliação e comparação dos métodos considerados:

1) A relação sinal-ruído média $\overline{S N R}$ representa a diferença média entre os $N_{h}$ picos harmônicos $H_{q}(q=$ $\left.1,2, \ldots, N_{h}\right)$ e o nível médio de ruído de fundo $A N L$. $\overline{S N R}$ indica o grau de resiliência ao ruído de fundo e é dada por:

$$
\overline{S N R}=\frac{1}{N_{h}} \sum_{q=1}^{N_{h}} S N R_{q}=\frac{1}{N_{h}} \sum_{q=1}^{N_{h}}\left|H_{q}-A N L\right|
$$


2) A largura espectral média $\overline{\Delta F_{3 d B}}$, medida a $-3 \mathrm{~dB}$ abaixo dos valores de pico dos componentes, indica a resolução de frequência e é calculada pela Eq. 5:

$$
\overline{\Delta F_{3 d B}}=\frac{1}{N_{h}} \sum_{q=1}^{N_{h}}\left|F_{q 1}-F_{q 2}\right|
$$

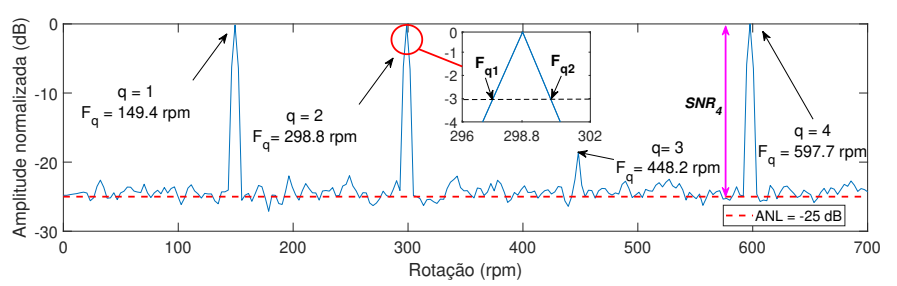

Fig. 5: Espectro de amplitude de um sinal de teste SONAR genérico, indicando os parâmetros gráficos $\overline{\Delta F_{3 d B}}$ e $\overline{S N R}$.

A análise das Fig.6a e Fig.6b mostra que, no caso da análise DEMON padrão (Fig.6a), as linhas espectrais do espectrograma são mais retilíneas do que as do HS obtido com a HHT-UPEMD (Fig.6b), enquanto a PSD média $\overline{P(f)}$ apresenta um menor espalhamento espectral do que o MHS. Este comportamento se deve, provavelmente, à elevada incidência de ruído no sinal experimental analisado, associada à grande sensibilidade ao ruído dos métodos baseados na EMD, entre os quais, a HHT-UPEMD.

No entanto, a análise das medidas de desempenho (Tab.II) revela aspectos não observados na abordagem visual anterior. Ao apresentar $\overline{S N R}=53.5 \mathrm{~dB}$, valor cerca de 7.5 vezes maior que o da DEMON padrão, a HHT-UPEMD é potencialmente mais resiliente ao ruído de fundo. Além disso, o valor $\overline{\Delta F_{3 d B}}=$ $2.4 \mathrm{rpm}$, apresentado pela HHT-UPEMD, é menos que a metade daquele apresentado pela DEMON padrão, concluindose, portanto, que a HHT-UPEMD possui uma maior resolução espectral. Neste sentido, tendo por base a HHT-UPEMD, o método proposto neste trabalho visa melhorar a estimação espectral da HHT para sinais ruidosos de SONAR passivo.
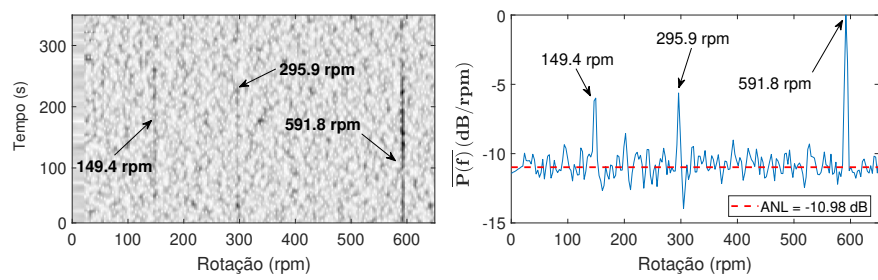

(a)
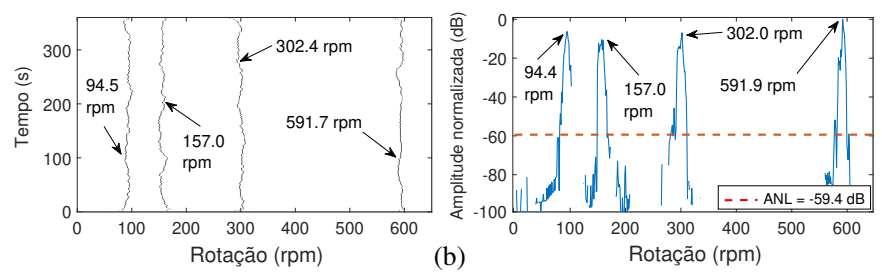

Fig. 6: Resultados dos métodos aplicados ao sinal experimental da Baía de Guanabara: (a) Análise DEMON padrão (espectrograma à esquerda e $\overline{P(f)}$ à direita), (b) HHT-UPEMD (HS à esquerda e MHS à direita).

Seguindo a metodologia proposta na Seção III, definiu-se o valor do limiar ótimo de classificação das IMFs $\left(\Delta_{L}\right)$, a ser usado na seleção das IMF-T e descarte das IMF-R (Fig.3). Os resultados da aplicação do método ao sub-conjunto de desenvolvimento (40 sinais-teste), são apresentados na Fig.7.

A análise consistiu em comparar-se as curvas ROC relativas às cinco configurações de parâmetros indicadas na Fig.7b, configurações essas utilizadas para a estimação da ACF. Em todas elas, usou-se uma janela retangular, $\mathrm{OL}=0.7$ e $\mathrm{DF}=$ 0.6. A configuração escolhida como a de melhor desempenho foi a que apresenta o ponto de operação com menor valor de FPR, uma vez fixado TPR $=90 \%$. Como pode ser visto, tal ponto corresponde ao $\mathrm{LAG}=[10: 250]$ e WS $=2048$, sendo, neste caso, FPR $=16 \%$. A partir das coordenadas deste ponto, obteve-se o valor otimizado de $\Delta_{L}=0.025$. Na Fig.7a, é mostrado o histograma da distribuição de valores $\Delta_{A C F}$ relativos à configuração que propiciou o melhor desempenho, indicando o limiar $\Delta_{L}$ otimizado.
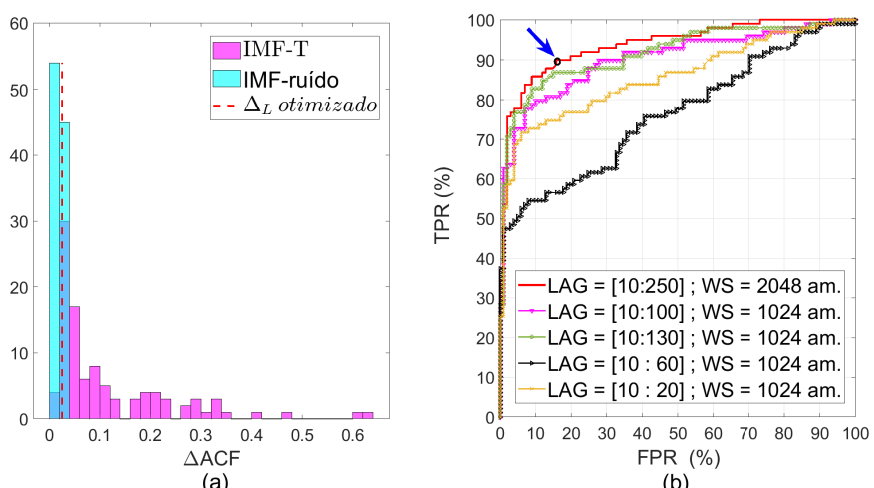

(b)

Fig. 7: Determinação do limiar de classificação das IMFs $\left(\Delta_{L}\right)$ (a) Distribuição de $\Delta_{A C F}$ das IMFs; (b) Curva ROC para cinco faixas de $\mathrm{LAG}$, indicando o ponto de operação mais eficiente (TPR=90\% e FPR $=16 \%$ ).

TABELA I: Resultados da validação do limiar de classificação $\left(\Delta_{L}\right)$.

\begin{tabular}{|c||c|c|c|c|c|c|}
\hline LAG & WS & $\Delta_{L}$ & $\begin{array}{c}\text { FPR } \\
\text { Valid. } \\
(\%)\end{array}$ & $\begin{array}{c}\text { TPR } \\
\text { Valid. } \\
(\%)\end{array}$ & $\begin{array}{c}\text { FPR } \\
\text { Desenv. } \\
(\%)\end{array}$ & $\begin{array}{c}\text { TPR } \\
\text { Desenv. } \\
(\%)\end{array}$ \\
\hline \hline$[10: 250]$ & 2048 & 0.025 & 27.3 & 96.4 & 16.0 & 90.0 \\
\hline$[10: 100]$ & 1024 & 0.031 & 36.0 & 93.0 & 28.0 & 90.0 \\
\hline$[10: 130]$ & 1024 & 0.025 & 31.8 & 96.4 & 35.0 & 90.0 \\
\hline$[10: 60]$ & 1024 & 0.032 & 77.3 & 92.9 & 64.0 & 90.0 \\
\hline$[10: 20]$ & 1024 & 0.039 & 72.7 & 85.7 & 70.0 & 90.0 \\
\hline
\end{tabular}

A validação do valor de $\Delta_{L}$ obtido na etapa de desenvolvimento foi realizada aplicando-se a HHT-modificada ao subconjunto de validação, composto por 10 sinais-teste pertencentes ao mesmo conjunto de dados, porém distintos dos 40 sinais-teste usados naquela etapa.

$\mathrm{Na}$ Tab.I, comparando as TPR nos conjuntos de validação e desenvolvimento (mantendo o $\Delta_{L}$ de desenvolvimento), verificamos que, em sua maioria, as primeiras são maiores. Quanto às FPR na validação, elas superam, em média, os valores do desenvolvimento. Este resultado mostra uma sensibilidade ao valor do $\Delta_{L}$ para diferentes sinais de SONAR passivo. Em uma aplicação prática, o valor do limiar pode ser ajustado pelo operador ara atender às necessidades do cenário em questão.

Por fim, é apresentado na Fig.8, o resultado da etapa de classificação das IMFs, para o sinal experimental de SONAR passivo adquirido na Baía de Guanabara, usando a configuração de parâmetros que apresentou o melhor desempenho. 
Pode-se observar que as $\operatorname{IMF}(2,3,4)$, contendo componentes harmônicos significativos, apresentam valores de $\Delta_{A C F}$ maiores que $\Delta_{L}=0.025$, sendo classificadas como IMF-T. Já a IMF1, contendo apenas ruído, apresenta um valor de $\Delta_{A C F}$ inferior a esse limiar, sendo classificada com êxito, como IMF-R. No caso da IMF5, apesar de não conter componentes harmônicos significativos, apresenta $\Delta_{A C F}>\Delta_{L}$, o que a classifica incorretamente como IMF-T (falso positivo).

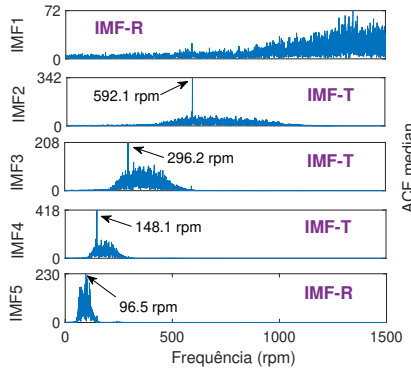

(a)

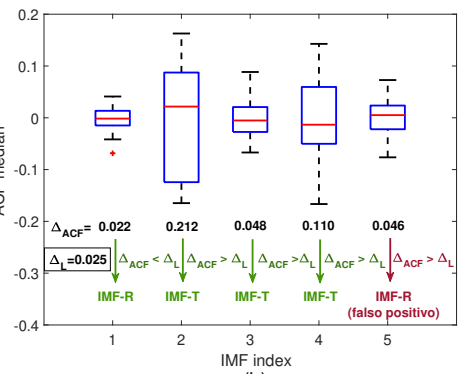

(b)
Fig. 8: Resultados da classificação das IMFs do sinal experimental da Baía de Guanabara: (a) IMFs; (b) Distribuição das medianas dos coeficientes ACF de cada IMF, indicando os valores dos intervalos interquartil $\triangle_{A C F}$.

Em seguida à etapa de seleção das IMFs, a aplicação da WTD (mesmo considerando a IMF5 - falso positivo) reduziu notadamente o nível de ruído, sem perda significativa de informação. Quando comparados à Fig.6b, o HS e o MHS resultantes da aplicação do WTD (Fig.9) apresentam linhas espectrais mais retilíneas e menos espalhamento espectral, respectivamente. Nesta análise, foram usados a função wavelet Daubechies 4 (db4) e quatro níveis de decomposição, parâmetros estes escolhidos empiricamente.

Analisando os valores das medidas de desempenho ao se aplicar a WTD (Tab. II), verificamos um aumento de $25 \mathrm{~dB}$ na $\overline{S N R}$ e uma diminuição de $80 \%$ em $\overline{\Delta F_{3 d B}}$, comparandose aos valores originais (53.5 dB e $2.4 \mathrm{rpm}$, respectivamente), ainda permanecendo melhores que os da DEMON padrão (Fig.6a). O desvio percentual médio $\overline{\Delta F_{p}}$ (considerando-se apenas os harmônicos das três IMF-T) entre a estimativa de frequência da HHT-UPEMD-WTD e a da análise DEMON foi de $0.4 \%$, valor este seis vezes menor que o da HHT-UPEMD. Discrepâncias desta ordem de grandeza não afetam consideravelmente o processo de classificação das embarcações.

TABELA II: Valores das medidas de desempenho dos métodos aplicados ao sinal experimental da Baía de Guanabara.

\begin{tabular}{|c||c|c|c|}
\hline Método & $\overline{\Delta F_{p}}(\%)$ & $\overline{S N R}(\mathrm{~dB})$ & $\overline{\Delta F_{3 d B}}(\mathrm{rpm})$ \\
\hline \hline DEMON padrão & - & 7.1 & 5.7 \\
\hline HHT-UPEMD & 2.4 & 53.5 & 2.4 \\
\hline HHT-UPEMD-WTD & 0.4 & 78.5 & 0.5 \\
\hline
\end{tabular}

\section{CONCLUSÕES}

Neste trabalho, foi apresentada uma versão modificada da HHT que visa melhorar o desempenho na estimação espectral em sinais de SONAR passivo. Uma etapa de eliminação das IMF contendo apenas ruído, utilizando os coeficientes da ACF, foi adicionada. Além disso, foi proposta uma filtragem WTD para as IMFs que tem informação relevante. Resultados com sinais experimentais mostram a eficiência do método proposto.
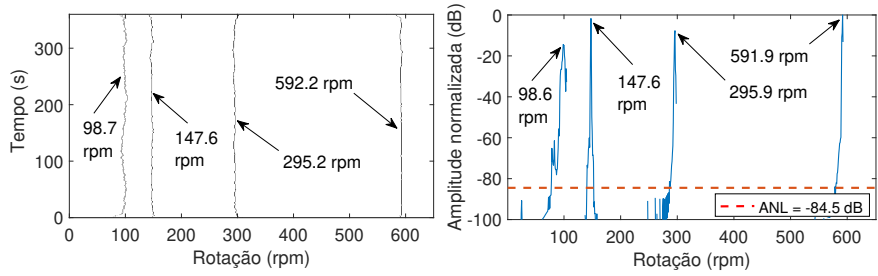

Fig. 9: Resultados do método proposto (HHT-modificada) aplicado ao sinal experimental da Baía de Guanabara (HS à esquerda and MHS à direita).

\section{AGRADECIMENTOS}

Os autores agradecem à FAPESB, CAPES, FAPERJ e CNPq pelo apoio financeiro e também ao Instituto de Pesquisas da Marinha do Brasil (IPqM) e à Sonitum - AtlantTIC (Universidade de Vigo, Espanha) pelo acesso aos dados experimentais.

\section{REFERÊNCIAS}

[1] P. C. Etter, Underwater acoustic modeling and simulation, 5th ed. CRC press, 2018

[2] A. V. Oppenheim and R. W. Schafer, Discrete-time signal processing, 3rd ed. Pearson Education India, 2014.

[3] N. N. de Moura, J. M. de Seixas, and R. Ramos, "Passive SONAR signal detection and classification based on independent component analysis," SONAR Systems, pp. 93-104, 2011.

[4] R. O. Nielson, Sonar signal processing. Artech House Inc, 1991.

[5] M. K. I. Molla and K. Hirose, "Hilbert spectrum in time-frequency representation of audio signals considering disjoint orthogonality," Advances in Adaptive Data Analysis, vol. 2, no. 03, pp. 313-336, 2010.

[6] N. E. Huang, Z. Shen, S. R. Long, M. C. Wu, H. H. Shih, Q. Zheng, N. C. Yen, C. C. Tung, and H. H. Liu, "The empirical mode decomposition and the Hilbert spectrum for nonlinear and non-stationary time series analysis," Proc. R. Soc. Lond. Ser. A, vol. 454, pp. 903-995, 1998.

[7] S. Zhang, L. Zhi, and T. Zhou, "Medical image retrieval using empirical mode decomposition with deep convolutional neural network," BioMed Research International, vol. 2020, pp. 1-12, 122020.

[8] H. Yadav, Y. Pal, and M. M. Tripathi, "Short-term pv power forecasting using empirical mode decomposition in integration with backpropagation neural network," Journal of Information and Optimization Sciences, vol. 41, pp. 25 - 37, 2020.

[9] Y. Zhang, J. C. Ji, and B. Ma, "Fault diagnosis of reciprocating compressor using a novel ensemble empirical mode decompositionconvolutional deep belief network," Measurement, vol. 156, 2020.

[10] L. Zao and R. Coelho, "Empirical signal decomposition for acoustic noise detection," IEEE Sensor Array and Multichannel Signal Processing Workshop (SAM), pp. 1-5, 2016.

[11] Y. Li, L. Yaan, X. Chen, J. Yu, H. Yang, and L. Wang, "A new underwater acoustic signal denoising technique based on ceemdan, mutual information, permutation entropy and wavelet threshold denoising," Entropy (Basel, Switzerland), vol. 20(8), p. 563, 2018.

[12] A. Lerch, An introduction to audio content analysis: Applications in signal processing and music informatics. Wiley-IEEE Press, 2012.

[13] D. L. Donoho and I. M. Johnstone, "Ideal spatial adaptation by wavelet shrinkage," Biometrika, vol. 81, no. 3, pp. 425-455, 1994.

[14] M. A. Colominas, G. Schlotthauer, M. E. Torres, and P. Flandrin, "Noiseassisted EMD methods in action," Advances in Adaptive Data Analysis, vol. 4, no. 04, 2012.

[15] Y. Wang, K. Hu, and M. Lo, "Uniform phase empirical mode decomposition: An optimal hybridization of masking signal and ensemble approaches," IEEE Access, vol. 6, pp. 34819-34 833, 2018.

[16] IPqM. (accessed: 05.11.2018) Etas 2018 contest dataset, as part of the XIII brazilian underwater acoustics technology meeting, rio de janeiro, brazil, 2018. [Online]. Available: https://www.marinha.mil.br/ipqm/etas

[17] D. Santos-Domínguez, S. Torres-Guijarro, A. Cardenal-López, and A. Pena-Gimenez, "Shipsear: An underwater vessel noise database," Applied Acoustics, vol. 113, pp. 64-69, 2016. 